Military Technical College Kobry El-Kobbah, Cairo, Egypt.

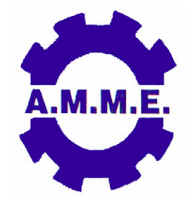

$15^{\text {th }}$ International Conference on Applied Mechanics and Mechanical Engineering.

\title{
ON STEADY MHD FLOW AND HEAT TRANSFER OF A NON-NEWTONIAN FLUID ABOVE A ROTATING POROUS DISK WITH ION SLIP AND OHMIC HEATING
}

\author{
H. A. Attia*, M. E. S. Ahmed ${ }^{* *}$ K. M. Ewis ${ }^{* \star *}$ and I. Hamdy ${ }^{* \star}$
}

\begin{abstract}
The steady flow of an electrically conducting non-Newtonian fluid due to the rotation of an infinite porous disk is studied with heat transfer with the inclusion of the ion slip as well as Ohmic heating. An external uniform magnetic field is applied perpendicular to the disk while a uniform suction or injection is imposed through the porous disk. The governing nonlinear momentum equations and energy equations are solved using the finite difference method. The results indicate the important effect of the ion slip and the non-Newtonian fluid characteristics on the velocity and temperature distributions.
\end{abstract}

\section{KEY WORDS}

Non-Newtonian fluids, Rotating disk flow, hydromagnetic flow, heat transfer, numerical solution, finite differences.

Professor, Dept. of Engineering Mathematics and Physics, Fayoum University, ElFayoum-63514, Egypt.

** Professor, Dept. of Engineering Mathematics and Physics, Fayoum University, ElFayoum-63514, Egypt.

*** Lecturer, Dept. of Engineering Mathematics and Physics, Fayoum University, ElFayoum-63514, Egypt. 


\section{NOMENCLATURE}

$\begin{array}{ll}a \ldots & \text { constant } \\ B_{0} \ldots & \text { a constant flux density } \\ B e \ldots & \text { the Hall parameter } \\ B i \ldots & \text { the ion slip parameter } \\ c_{p} \ldots & \text { the specific heat capacity } \\ E \ldots \ldots & \text { the Eckert number } \\ F, G, H \ldots & \text { non-dimensional function } \\ K \ldots & \text { the parameter which describes the non-Newtonian behavior } \\ k \ldots & \text { the thermal conductivity of the fluid. } \\ n \ldots & \text { the electron concentration per unit volume } \\ P r \ldots & \text { the Prandtl number } \\ p \ldots & \text { the pressure } \\ R e \ldots & \text { the Reynolds number } \\ T \ldots & \text { the temperature } \\ u \ldots & \text { the fluid velocity in direction } r \\ V \ldots & \text { the fluid velocity in direction } \varphi \\ w \ldots & \text { the fluid velocity in direction } z \\ \omega \ldots & \text { a uniform angular velocity } \\ \rho \ldots & \text { the density of the fluid } \\ \sigma \ldots & \text { the fluid electrical conductivity } \\ \beta \ldots & \text { the Hall factor } \\ \zeta \ldots & \text { a non-dimensional distance measured along the axis of rotation } \\ \mu \ldots & \text { the viscosity of the fluid } \\ \mu_{C} \ldots & \text { the coefficient of cross viscosity } \\ V \ldots & \text { the magnetic interaction number }\end{array}$

\section{INTRODUCTION}

The flow due to an infinite rotating disk was studied by von Karman [1] who formulated the problem in the steady state and introduced similarity transformations to transform the governing nonlinear partial differential equations to nonlinear ordinary differential equations. Asymptotic solutions for the resulting nonlinear ordinary differential equations were given by Cochran [2]. Rotating disk flows of an electrically conducting fluids have interesting applications in many areas such as rotating machinery, lubrication, oceanography, computer storage devices, viscometry and crystal growth processes. The effect of an externally applied uniform magnetic field on the flow due to a rotating disk was examined in $[3,4]$. In the above mentioned work, the Hall and ion slip effects were ignored in applying Ohm's law, as their effect can be ignored for small and moderate values of the magnetic field $[5,6]$. The influence of the Hall current and ion slip on the steady hydromagnetic flow with heat transfer due to an infinite rotating was studied [7,8]. The effect of uniform suction or injection on the flow of an electrically conducting fluid due to a rotating disk was studied taking the Hall current [9] and the ion slip [10] into consideration. 
The problem of steady state heat transfer from a rotating disk at a constant temperature was examined by Millsaps and Pohlhausen [11] for a variety of Prandtl number. Sparrow and Gregg [12] studied the steady state heat transfer from a rotating disk maintained at a constant temperature to fluids at any Prandtl number. Attia [13] solved the unsteady state heat transfer in the presence of an applied uniform magnetic field. The steady flow of a non-Newtonian fluid above an infinite rotating disk with uniform suction was studied by Mithal [14]. The influence of a uniform magnetic field on the flow of the non-Newtonian fluid was studied by Attia [15].

In the present paper, the steady hydromagnetic flow of a viscous, incompressible, and electrically conducting non-Newtonian fluid due to the uniform rotation of an infinite disk in an axial uniform magnetic field is studied considering the ion slip and the Ohmic heating. The governing non-linear partial differential equations are solved numerically using the finite difference method. Some interesting effects for the magnetic field, the Hall current, the ion slip, and the non-Newtonian fluid characteristics on the velocity and temperature fields are discussed.

\section{BASIC EQUATIONS}

The disk is assumed to be electrically insulating and rotating impulsively from rest in the $z=0$ plane about the $z$-axis with a uniform angular velocity $\omega$. The fluid is assumed to be electrically conducting incompressible and has density $\rho$, kinematical viscosity $v$, and electrical conductivity $\sigma$. An external axial uniform magnetic field is applied in the $z$-direction and has a constant flux density $\boldsymbol{B}_{0}$. The magnetic Reynolds number is assumed to be very small, and therefore the induced magnetic field is negligible. The electron-atom collision frequency is assumed to be high, then both the Hall effect and the ion slip effects are taken into consideration $[5,6]$. Due to the axial symmetry about the $z$-axis, the cylindrical coordinates $(r, \varphi, z)$ are used. For the sake of definiteness, the disk is taken to be rotating in the positive $\varphi$ direction. Due to the symmetry about the $z=0$ plane, it is sufficient to consider the problem in the upper half space only. If the Hall and ion slip terms are retained in generalized Ohm's law, then the equations of steady motion in cylindrical coordinates $[5,6]$

$$
\begin{aligned}
& \frac{\partial u}{\partial r}+\frac{u}{r}+\frac{\partial w}{\partial z}=0 \\
& \rho\left(u \frac{\partial u}{\partial r}+w \frac{\partial u}{\partial z}-\frac{v^{2}}{r}\right)+\frac{\sigma B_{o}^{2}}{\left(\alpha^{2}+B e^{2}\right)}(\alpha u-B e v)=\frac{\partial \tau_{r}^{r}}{\partial r}+\frac{\partial \tau_{r}^{z}}{\partial z}+\frac{\tau_{r}^{r}-\tau_{\varphi}^{\varphi}}{r} \\
& \rho\left(u \frac{\partial v}{\partial r}+w \frac{\partial v}{\partial z}+\frac{u v}{r}\right)+\frac{\sigma B_{o}^{2}}{\left(\alpha^{2}+B e^{2}\right)}(\alpha v+B e u)=\frac{\partial \tau_{\varphi}^{r}}{\partial r}+\frac{\partial \tau_{\varphi}^{z}}{\partial z}+\frac{2 \tau_{\varphi}^{r}}{r} \\
& \rho\left(u \frac{\partial w}{\partial r}+w \frac{\partial w}{\partial z}\right)=\frac{\partial \tau_{z}^{r}}{\partial r}+\frac{\partial \tau_{z}^{z}}{\partial z}+\frac{\tau_{z}^{r}}{r}
\end{aligned}
$$


where $u, v$, and $w$ are the velocity components in the directions of increasing $r, \varphi$, and $z, B e=\sigma \beta B_{0}$ is the Hall parameter which can take positive or negative values, $\beta=1 / n q$ is the Hall factor, $n$ is the electron concentration per unit volume, $-q$ is the charge of the electron $[5,6], \alpha=1+B e B i$, and $B i$ is the ion slip parameter $[5,6]$.

The boundary conditions are given as

$$
\begin{aligned}
& z=0, u=0, v=r \omega, w=w_{o}, \\
& z \rightarrow \infty, u \rightarrow 0, v \rightarrow 0, p \rightarrow p_{\infty} .
\end{aligned}
$$

where $p$ is denoting the pressure. Due to the uniform suction or injection, the vertical velocity component takes a constant non-zero value at $z=0$. Introducing von Karman transformations [1],

$$
u=r \omega F, v=r \omega G, w=\sqrt{\omega v} H, z=\sqrt{v / \omega} \zeta, p-p_{\infty}=-\rho v \omega P
$$

where $\zeta$ is a non-dimensional distance measured along the axis of rotation, $F, G, H$ and $P$ are non-dimensional functions of the modified vertical coordinate $\zeta$, Eqs. (1)(5) take the form

$$
\begin{aligned}
& \frac{d H}{d \zeta}+2 F=0 \\
& \frac{d^{2} F}{d \zeta^{2}}-H \frac{d F}{d \zeta}-F^{2}+G^{2}-\frac{\gamma}{\left(\alpha^{2}+B e^{2}\right)}(\alpha F-B e G)-\frac{1}{2} K\left(\left(\frac{d F}{d \zeta}\right)^{2}+3\left(\frac{d G}{d \zeta}\right)^{2}+2 F \frac{d^{2} F}{d \zeta^{2}}\right)=0 \\
& \frac{d^{2} G}{d \zeta^{2}}-H \frac{d G}{d \zeta}-2 F G-\frac{\gamma}{\left(\alpha^{2}+B e^{2}\right)}(\alpha G+B e F)+K\left(\frac{d F}{d \zeta} \frac{d G}{d \zeta}-F \frac{d^{2} G}{d \zeta^{2}}\right)=0 \\
& \frac{d^{2} H}{d \zeta^{2}}-H \frac{d H}{d \zeta}-\frac{7}{2} K \frac{d H}{d \zeta} \frac{d^{2} H}{d \zeta^{2}}+\frac{d P}{d \zeta}=0 \\
& \zeta=0, F=0, G=1, H=0, \\
& \zeta \rightarrow \infty, F \rightarrow 0, G \rightarrow 0, P \rightarrow 0 .
\end{aligned}
$$

where $K$ is the parameter which describes the non-Newtonian behavior, $K=\mu_{d} d \mu \omega, \mu_{c}$ is the coefficient of cross viscosity, $V$ is the magnetic interaction number given by $\gamma=\sigma B_{0}{ }^{2} / \rho \omega$ which represents the ratio between the magnetic force to the fluid inertia force.

Due to the difference in temperature between the wall and the ambient fluid heat transfer takes place. The energy equation, neglecting the dissipation term, takes the form $[9,10]$,

$$
\rho c_{p}\left(u \frac{\partial T}{\partial r}+w \frac{\partial T}{\partial z}\right)=k\left(\frac{\partial^{2} T}{\partial z^{2}}+\frac{\partial^{2} T}{\partial r^{2}}+\frac{1}{r} \frac{\partial T}{\partial r}\right)+\frac{\sigma B_{o}^{2}}{\alpha^{2}+B e^{2}}\left(u^{2}+v^{2}\right)
$$

where $k$ and $c_{p}$ are, respectively, the thermal conductivity and the specific heat at constant pressure of the fluid. The last term in Eq. (11) represents the Ohmic 
heating. The boundary conditions for the energy problem are that the temperature, by continuity considerations, equals $T_{w}$ at the surface of the disk. Far from the disk, $T \rightarrow T_{\infty}$ where $T_{\infty}$ is the temperature of the ambient fluid. In terms of the nondimensional variable $\bar{T}=\left(T-T_{\infty}\right) /\left(T_{w}-T_{\infty}\right)$ and using von Karman transformations, Eq. (11) takes the form (the bar will be dropped),

$$
\frac{1}{\operatorname{Pr}} \frac{\partial^{2} T}{\partial \zeta^{2}}-H \frac{\partial T}{\partial \zeta}+\frac{\gamma E c}{\alpha^{2}+B e^{2}}\left(F^{2}+G^{2}\right)=0
$$

where $\operatorname{Pr}$ is the Prandtl number given by, $\operatorname{Pr}=c_{p} \mu / k$ and $E c=\omega^{2} r^{2} / c_{p}\left(T_{w}-T_{\infty}\right)$ is the Eckert number. The boundary conditions, in terms of $T$ and von Karman transformations, are expressed as

$\zeta=0: T=1, \zeta \rightarrow \infty, T \rightarrow 0$,

The heat transfer from the disk surface to the fluid is computed by application of Fourier's law

$q=-k\left(\frac{d T}{d z}\right)_{w}$

Introducing the transformed variables, the expression for $q$ becomes

$q=-k\left(T_{w}-T_{\infty}\right) \sqrt{\frac{\omega}{v}} \frac{d T(0)}{d \zeta}$

By rephrasing the heat transfer results in terms of a Nusselt number defined as,

$$
\begin{aligned}
& N_{u}=q \sqrt{v / \omega} / k\left(T_{w}-T_{\infty}\right) \text { the last equation becomes } \\
& N_{u}=-\frac{d T(0)}{d \zeta}
\end{aligned}
$$

The system of non-linear ordinary differential equations (6)-(8) is solved under the conditions given by Eq. (10) to determine the velocity distribution, using the CrankNicolson method [16]. Then Eq. (12) is solved under the boundary conditions (13) to find the temperature distribution. The resulting system of difference equations is solved in the infinite domain $0<\zeta<\infty$, by considering a finite domain in the $\zeta$-direction instead with $\zeta$ chosen large enough such that the solutions are not affected by imposing the infinite asymptotic conditions at a finite distance. The independence of the results from the chosen length of the implemented finite domain and the size of mesh used was tested by numerical experiments. Computations are carried out for $\zeta_{\infty}=10$ which is adequate for the ranges of the parameters studied here.

\section{RESULTS AND DISCUSSION}

Figures 1 a-d present the profile of the radial velocity component $F$ for various values of the Hall parameter $\mathrm{Be}$, the ion slip parameter $B i$, and the non-Newtonian 
parameter $K$ and for $\gamma=1$ and $K=0$ and 1 . Figure 1 indicates that for $B e=-0.5$ and $B i=0$, the radial flow reverses its direction at a certain distance from the surface of the disk. Increasing $B i$, for $B e<0$, reverses the direction of $F$ for all $\zeta$ as a result of increasing the effective conductivity $\left.\left(=\mathrm{y} /\left(\alpha^{2}+\mathrm{Be}^{2}\right)\right)\right)$ which increases the resistive force on $F$. Figure 1 a shows also that for $B e B i>0$, increasing the magnitude of $B i$ increases $F$ as a result of decreasing the effective conductivity which decreases the damping force on $F$. Large values of $B i$ decrease the effective conductivity more which is similar to the hydrodynamic case. Figure $1 \mathrm{~b}$ depicts that when $B e>0$ and $B i>0$, increasing $B i$ decreases $F$ for some $\zeta$. This is because the effect of $B i$ on the numerator in the magnetic force term in Eq. (7) is higher than its effect on the denominator which increases the damping force on $F$ and consequently decreases $F$ for some $\zeta$. Also, for $B i<0$, increasing the magnitude of $B i$ increases $F$ for small $\zeta$ and then decreases it for larger $\zeta$. This is corresponding to the crossover in the $F-\zeta$ chart with $B i$. It is interesting to find that the changing of $F$ with $B i$ depends on $\zeta$. The effect of the parameter $K$ is shown in Figs. $1 \mathrm{c}$ and $1 \mathrm{~d}$. The figures show that the effect of the parameter $K$ is to reverse the direction of $F$ for the case $B i=B e=0$. For $B e<0$, as shown in Fig. 1c, the parameter $K$ reverses the direction of $F$ for all values of $B i$. In Fig. 1d, for $B e>0, K$ reduces the magnitude of $F$ while keeping its direction where the value at which the crossover in the $F-\zeta$ charts occur increases with $K$.

Figures 2a-d present the profile of the azimuthal velocity component $G$ for various values of the Hall parameter $\beta_{e}$, the ion slip parameter $B i$ and the non-Newtonian parameter $K$ and for $\gamma=1$ and $K=0$ and 1. As shown in Fig. 2a, small negative values of $B e$ increases $G$ due to decreasing the magnetic damping force. Increasing $B i$, with $B e<0$, reduces $G$, as a result of increasing the effective conductivity. Figure $2 a$ indicated that for negative values of $B i$, increasing the magnitude of $B i$ increases $G$ as a result of decreasing the damping force on $G$. Figure $2 b$ depicts the same findings. For $B e B i>0$, increasing $B i$ increases $G$, on the other hand, for $B e B i<0$, increasing the magnitude of $B i$ decreases $G$. Figures $2 \mathrm{c}$ and $2 \mathrm{~d}$ indicate that increasing $K$ increases $G$ for all $B e$ and $B i$. The effect of $B i$ on $G$ is more pronounced for $K>0$.

Figures 3a-d present the profile of the axial velocity component $H$ for various values of the Hall parameter $B e$, the ion slip parameter $B i$, and the non-Newtonian parameter $K$ and for $\mathrm{y}=1$ and $K=0$ and 1 . As shown in Fig. $3 a$, for $B e=-0.5$ and $B i=0$, the axial velocity $H$ reverses its direction at a certain $\zeta$. Increasing $B i$ increases $H$, as a result of decreasing $F$, and reverses its direction for all $\zeta$. Figure $3 b$ indicates that for $B e>0$, increasing the magnitude of $B i$, in general, decreases $H$ as a result of increasing $F$. It is also shown in Fig. $3 \mathrm{~b}$ that the axial flow is always towards the disk for all values of $B i$. Figures $3 c$ and $3 d$ show the effect of the non-Newtonian parameter $K$ in reversing the direction of $H$ for the case $B e=B i=0$. For $B e<0$, as shown in Fig. 3c, the parameter $K$ reverses the direction of $H$ for all values of $B i$. In Fig. $3 \mathrm{~d}$, for $B e>0, K$ reduces the magnitude of $H$ while keeping its direction.

Figures $4 a-d$ present the profile of the temperature $T$ for various values of the Hall parameter $B e$, the ion slip parameter $B i$, and the non-Newtonian parameter $K$ and for $Y=1, P r=0.7$ and $K=0$ and 1 . It is shown in Figs. $4 a$ and $4 c$ that, for $B i>0$, increasing $B i$ increases $T$ as a result of increasing $H$ but for $B i<0$, increasing the magnitude of $B i$ decreases $T$ due to decreasing $H$. On the other hand, Figs. $4 b$ and $4 d$, shows that for $B i>0$, increasing $B i$ decreases $T$ as a result of decreasing $H$, however for $B i<0$, 
increasing the magnitude of $B i$ increases $T$ due to increasing $H$. It is shown in Fig. 4 that the effect of the ion slip parameter $B i$ on the temperature $T$ becomes more apparent for higher values of $K$ and for positive values of the Hall parameter than for negative values.

Tables 1 and 2 show the variation of the Nusselt number $N_{u}$ with the Hall parameter $B e$, the ion slip parameter $B i$, and the non-Newtonian parameter $K$ for $P r=0.7$ and 10, respectively. In these tables $\mathrm{y}=1$ and $E c=0.2$. It is shown that, for $B e<0$ and all values of $S$, increasing $B i$ decreases $N_{u}$ due to the decrease in the incoming axial flow at near-ambient temperature to the disk which decreases the heat transfer from the surface of the disk. On the other hand, for $B e>0$ and for all values of $S$, increasing $\mathrm{Bi}$ increases the incoming flow and then increases $N_{u}$. The influence of $\mathrm{Bi}$ on $N_{u}$ is more clear for $B e>0$ than for $B e<0$. Increasing the parameter $K$ reduces $N_{u}$ for all $B e, B i$ and $P r$. This is expected since the effect of the non-Newtonian behavior is to restrain the axial flow towards the disk and then prevent bringing the fluid at near-ambient temperature to the disk which decreases $N_{u}$.

\section{CONCLUSIONS}

The steady flow of an electrically conducting non-Newtonian fluid due to the rotation of an infinite disk in the presence of an externally applied uniform magnetic field is studied with heat transfer considering the Hall effect, the ion slip and the Ohmic heating. The inclusion of the Hall effect, the ion slip and the non-Newtonian fluid characteristics reveals some interesting phenomena and it is found that both the magnitudes and signs of the Hall and ion slip parameters are effective. It is interesting to find, in the Newtonian case, that the radial and axial flows reverse direction with the distance $\zeta$ for $B e<0$ and all values of $B i$. The effect of the nonNewtonian parameter $K$ is to reverse the axial and radial flow for $B e=B i=0$. Also, in the non-Newtonian case, both the axial and radial flows reverse direction for all $\zeta$ for $B e<0$ and all values of $B i$. For $B e>0$, the dependence of the velocity components in the ion slip changes with $\zeta$. The heat transfer at the surface of the disk is found to vary with the magnitude and sign of the Hall and ion slip parameters. The effect of $B i$ on the temperature as well as the heat transfer at the surface of the disk is more apparent for higher values of $K$ and for $B e<0$ than $B e>0$. On the other hand, the effect of non-Newtonian parameter $K$ on the heat transfer is more clear for $B e>0$ than $B e<0$.

\section{REFERENCES}

[1] von Karman, T., "Uber laminare und turbulente reibung", ZAMM, vol. 1, No. 4, pp. 233-235, (1921).

[2] Cochran, W.G., "The flow due to a rotating disk", Proc. Cambridge Philos. Soc. 30 (3), pp. 365-375, (1934).

[3] El-Mistikawy, T.M.A. and Attia, H.A., "The rotating disk flow in the presence of strong magnetic field", Proc. 3rd Int. Congress of Fluid Mechanics, Cairo, Egypt, vol. 3, pp. 1211-1222, Jan. 2-4, (1990). 
[4] El-Mistikawy, T.M.A., Attia, H.A., and Megahed, A.A., "The rotating disk flow in the presence of weak magnetic field", Proc. 4th Conference on Theoretical and Applied Mechanics, Cairo, Egypt, pp. 69-82, Nov. 5-7, (1991).

[5] Sutton, G.W. and Sherman, A., Engineering magnetohydrodynamics, McGraw-Hill, New York, (1965).

[6] Crammer, K.R. and Pai, S.-I., Magnetofluid dynamic for engineers and applied physicists, New York, McGraw-Hill, (1973).

[7] Aboul-Hassan, A.L. and Attia, H.A., "The flow due to a rotating disk with Hall effect", Physics Letters A, 228, pp. 286-290, (1997).

[8] Attia, H.A., "On the effectiveness of ion slip on steady MHD flow and heat transfer above a rotating disk with Ohmic heating", Journal of Fluids Engineering, Vol. 128, Issue 5, (2006).

[9] Attia, H.A. and Aboul-Hassan, A.L., "Effect of Hall current on the unsteady MHD flow due to a rotating disk with uniform suction or injection", Applied Mathematical Modelling, Vol. 25, No. 12, pp. 1089-1098, (2001).

[10] Attia, H.A., "On the effectiveness of ion slip and uniform suction or injection on steady MHD flow due to a rotating disk with heat transfer and ohmic heating", Chemical Engineering Communications (CEC), Vol. 194, Issue 10, pp. 1396-1407, (2007).

[11] Millsaps, K. and Pohlhausen, K., "Heat transfer by laminar flow from a rotating disk", J. of the Aeronautical Sciences, Vol. 19, pp. 120-126, (1952).

[12] Sparrow, E.M. and Gregg, J.L., "Mass transfer, flow, and heat transfer about a rotating disk", ASME J. of Heat Transfer, pp. 294-302, (1960).

[13] Attia, H.A., "Unsteady MHD flow near a rotating porous disk with uniform suction or injection", Fluid Dynamics Research 23, pp. 283-290, (1998).

[14] Mithal, K.G., "On the effects of uniform high suction on the steady flow of a non-Newtonian liquid due to a rotating disk", Quart. J. Mech. Appl. Math., Vol. XIV, pp. 401-410, (1961).

[15] Attia, H.A., "Unsteady flow of a non-Newtonian fluid above a rotating disk with heat transfer", Int. J. of Heat and Mass Transfer, Vol. 46, No. 41, pp. 26952700, (2003).

[16] Ames, W.F., Numerical methods in partial differential equations, 2nd ed., Academic Press, New York, (1977). 
FIGURES AND TABLES:

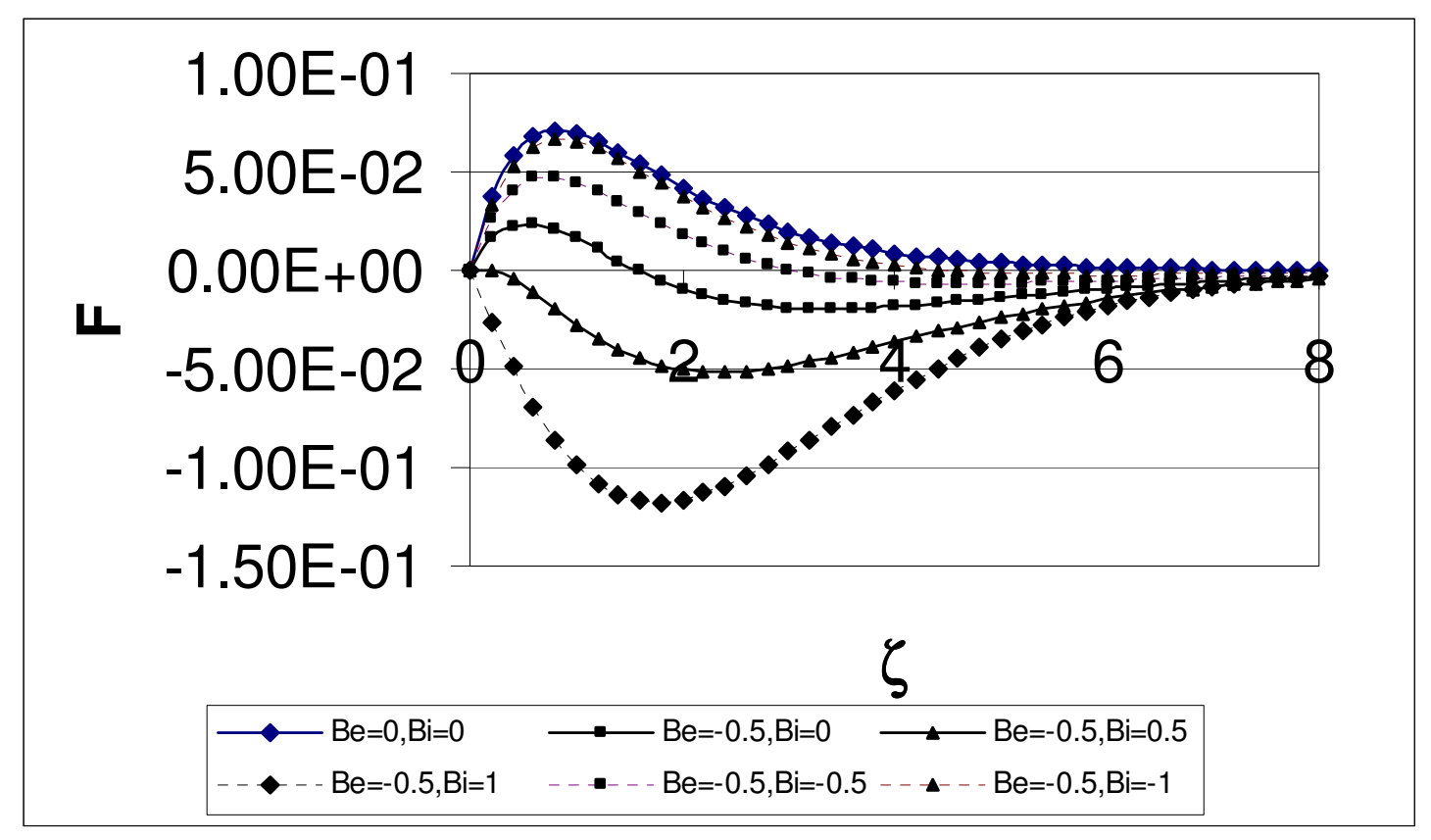

(a)

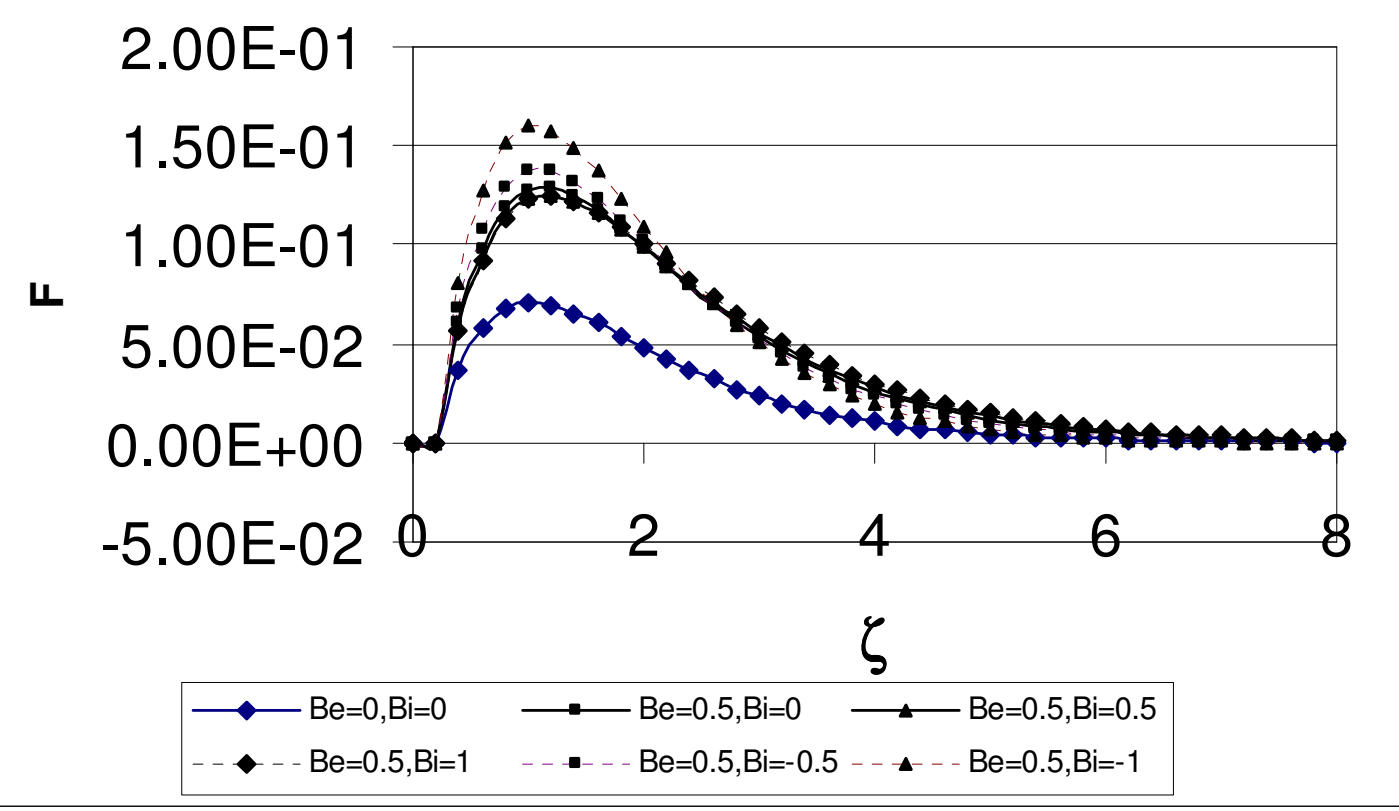

(b) 


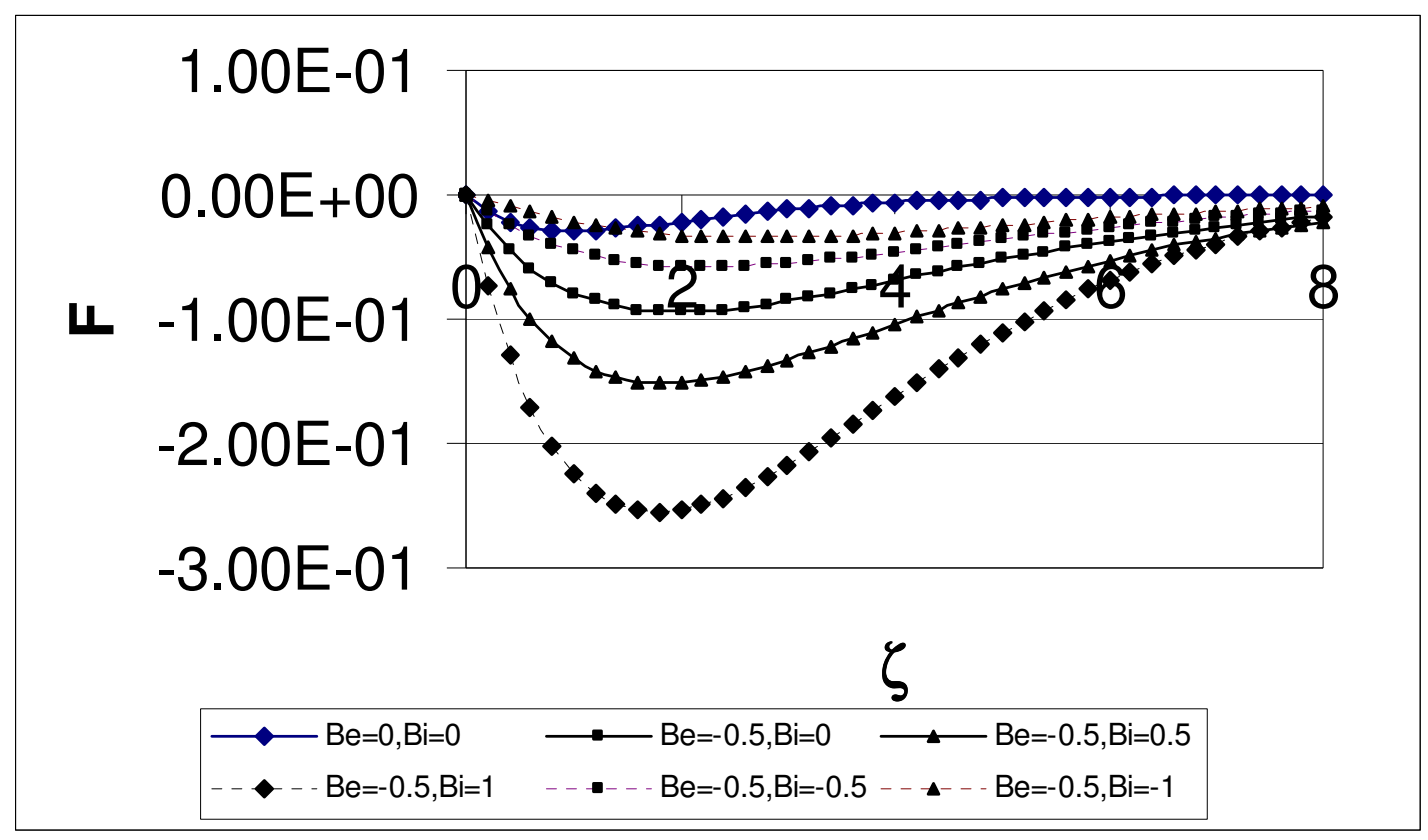

(c)

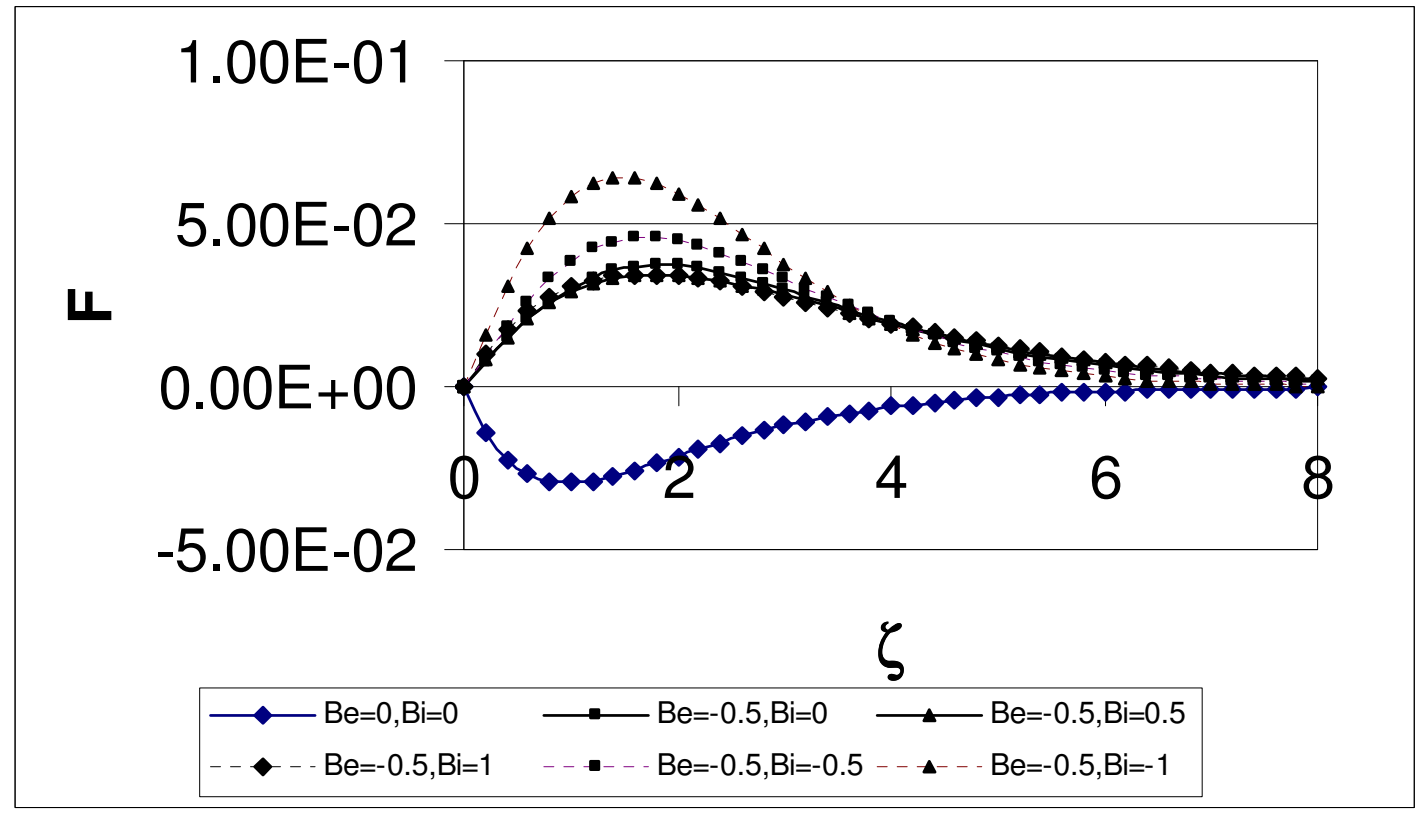

(d)

Fig. 1 Steady state profile of $F$ for various values of $B e, B i$ and $K$. (a) $K=0, B e \leq 0$;

(b) $\mathrm{K}=0, \mathrm{Be} \geq 0$; (c) $\mathrm{K}=1$, $\mathrm{Be} \leq 0$; and (d) $\mathrm{K}=1, \mathrm{Be} \geq 0$. 


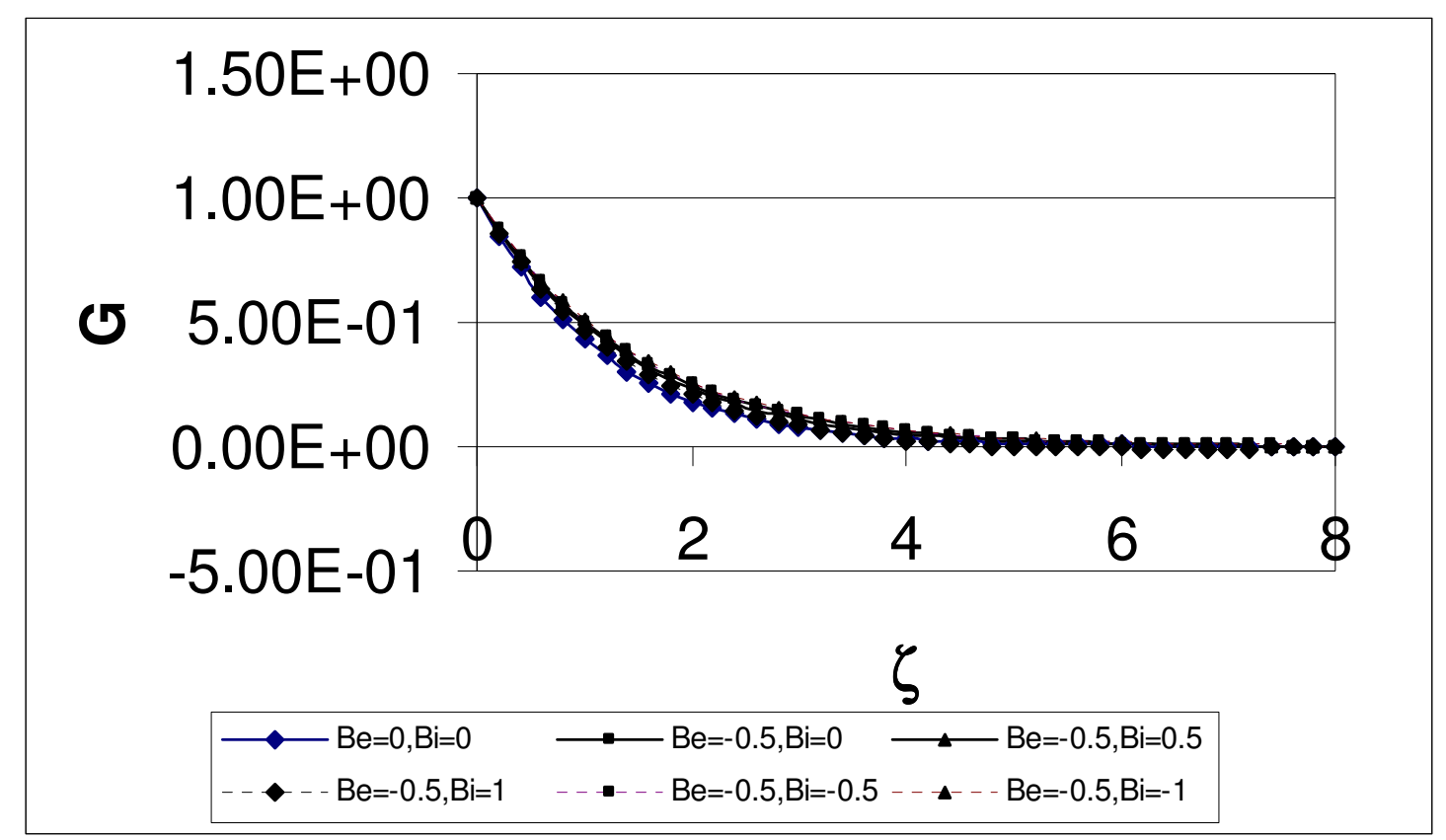

(a)

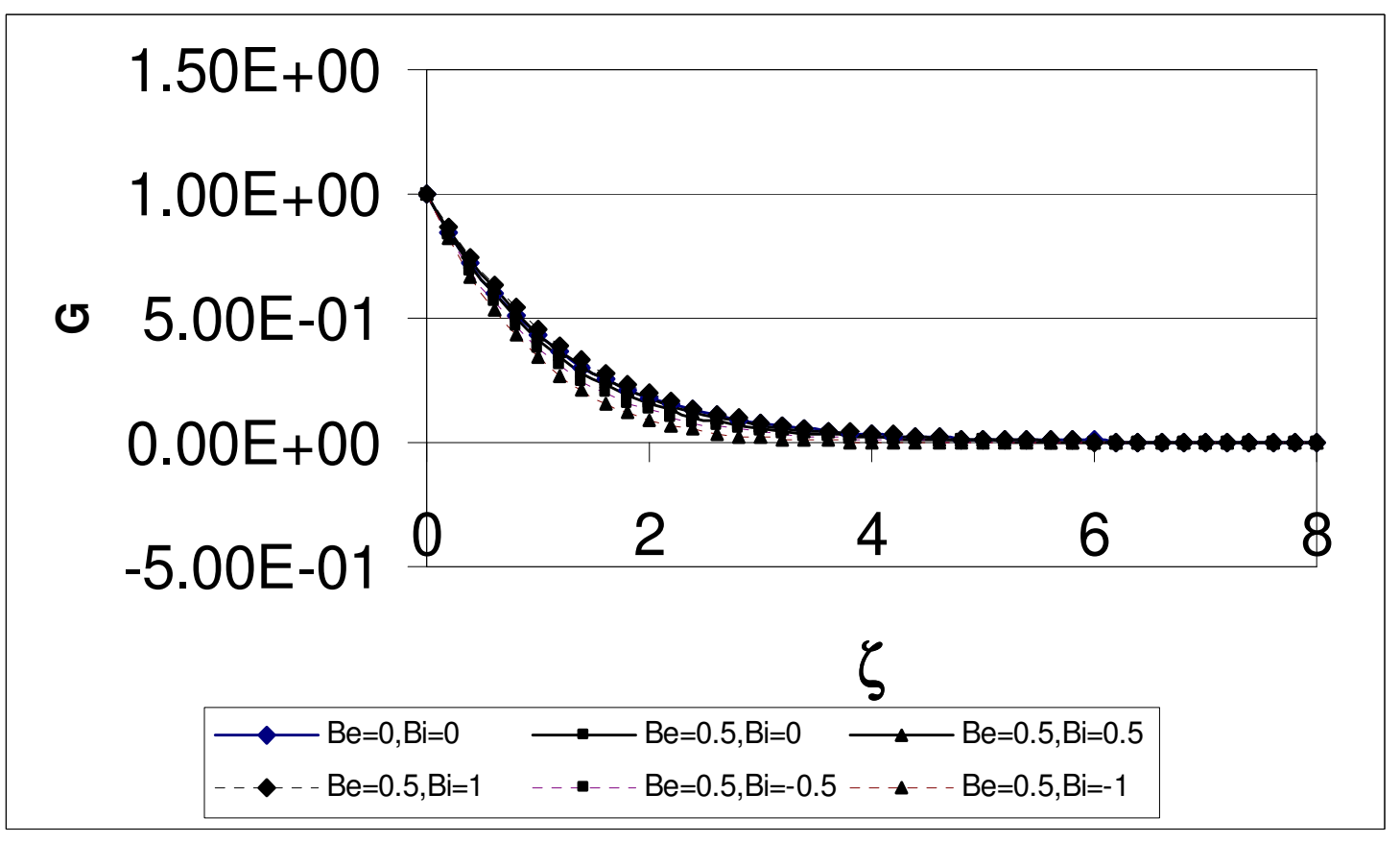

(b) 


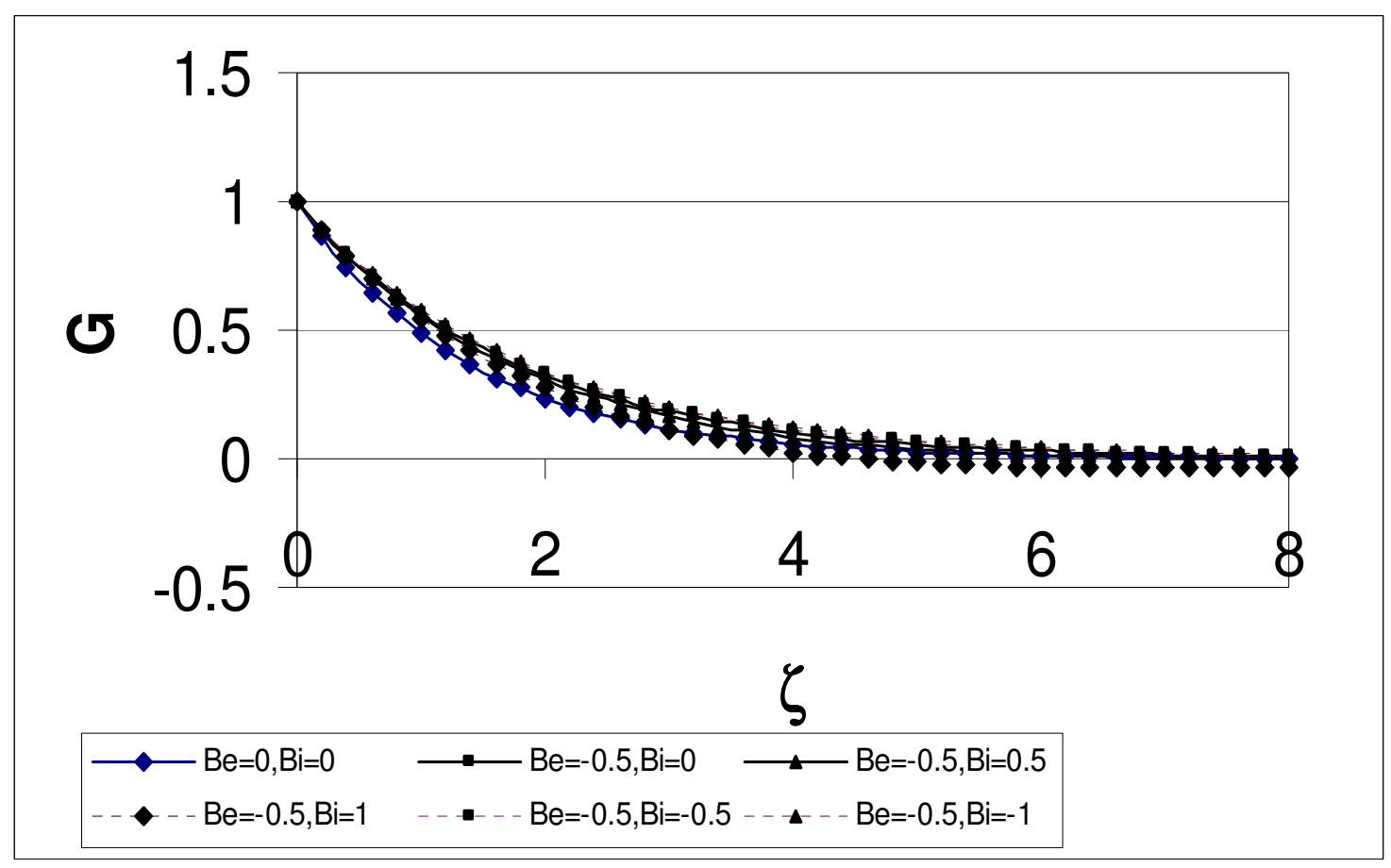

(c)

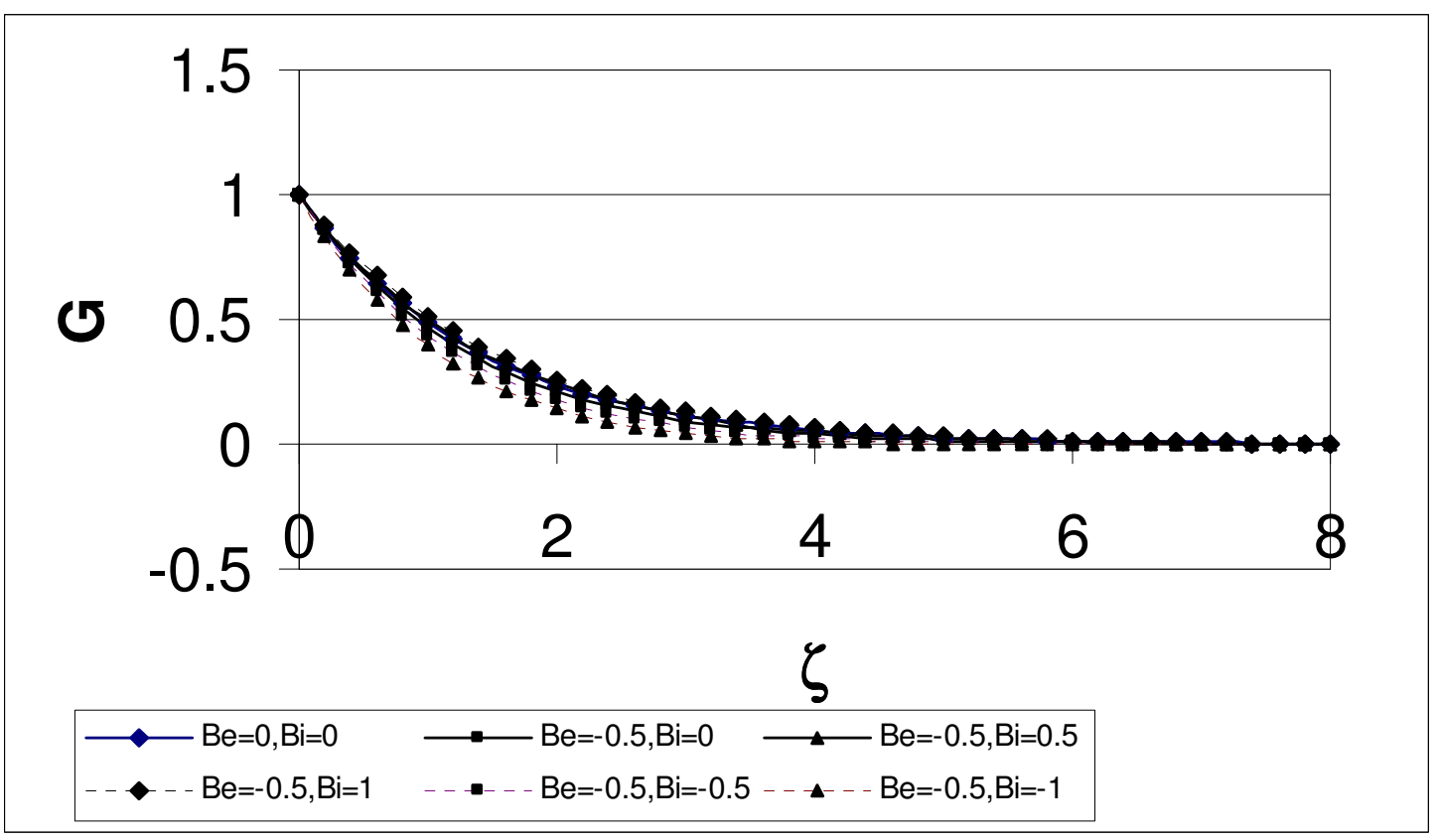

(d)

Fig. 2 Steady state profile of $G$ for various values of $B e, B i$ and $K$. (a) $K=0, B e \leq 0$; (b) $\mathrm{K}=0, \mathrm{Be} \geq 0$; (c) $\mathrm{K}=1, \mathrm{Be} \leq 0$; and (d) $\mathrm{K}=1, \mathrm{Be} \geq 0$. 


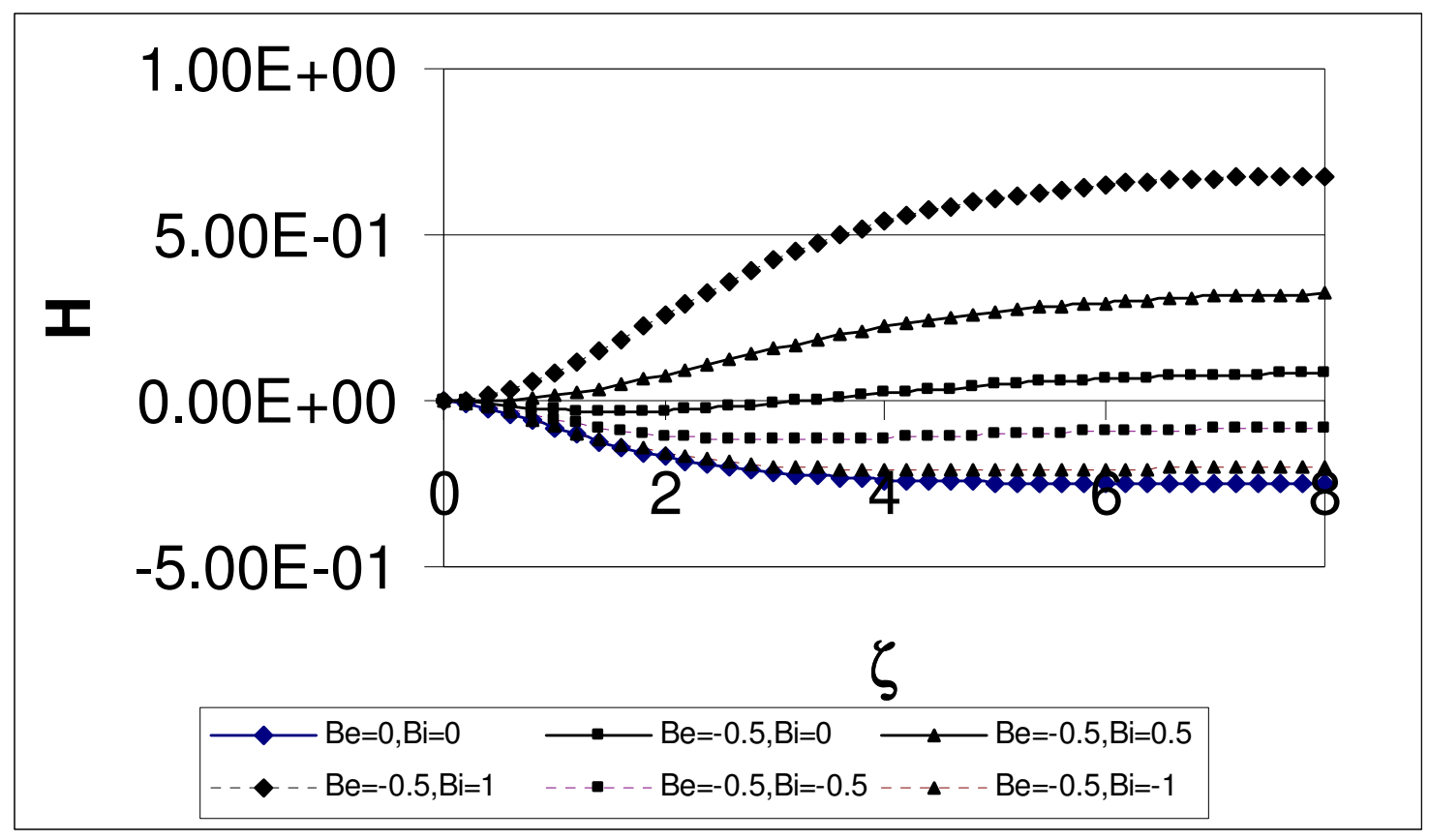

(a)

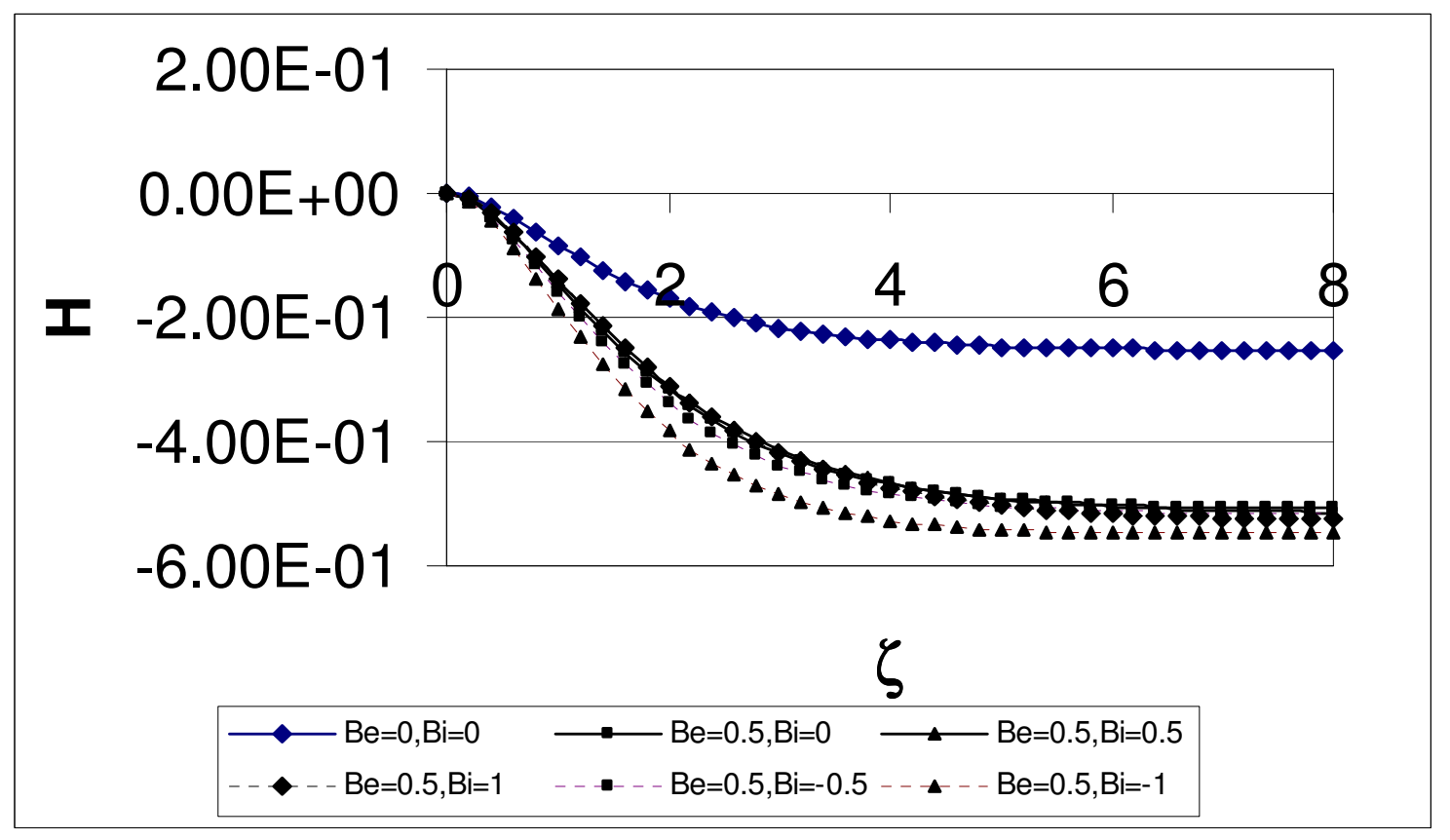

(b) 


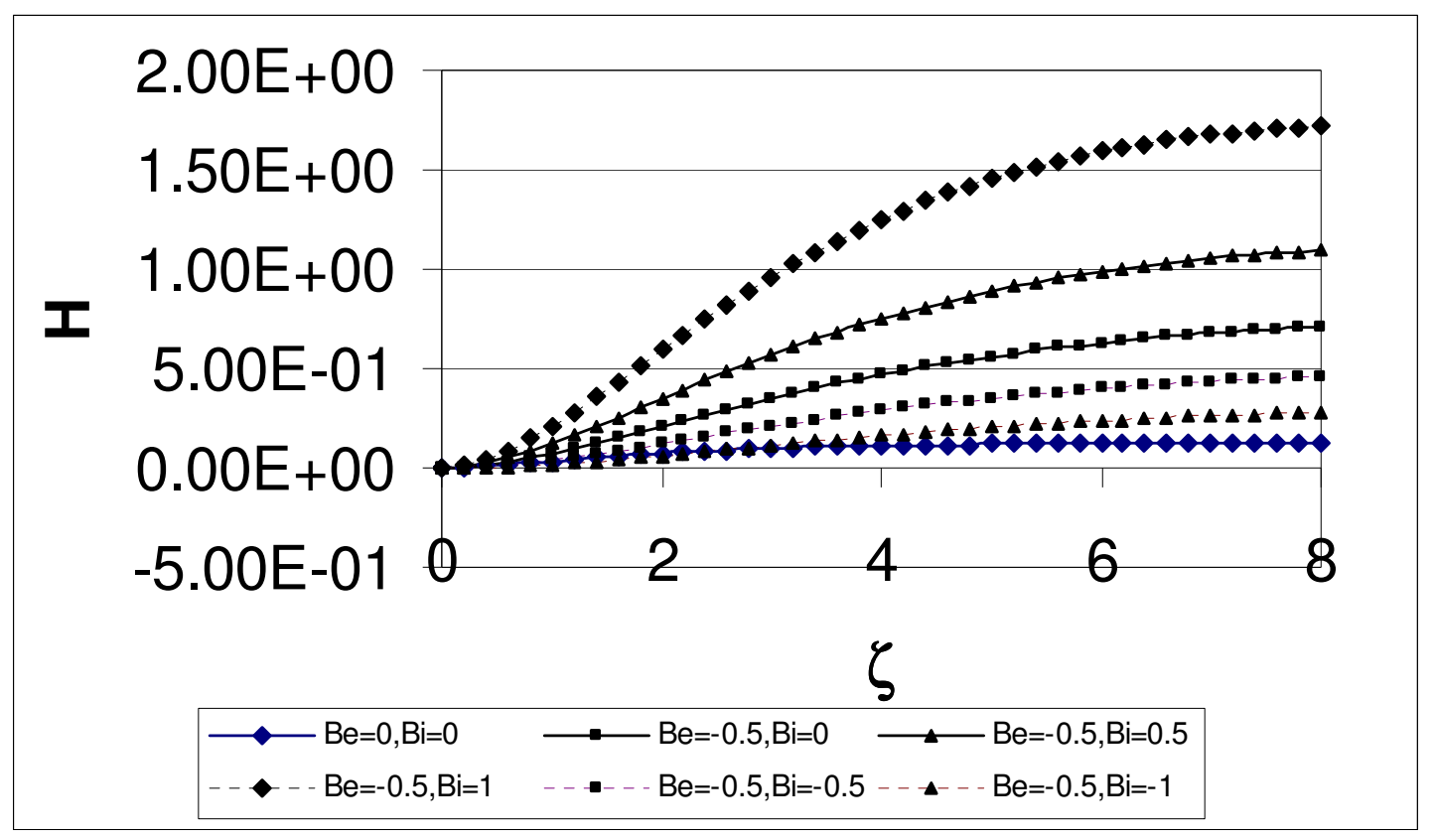

(c)

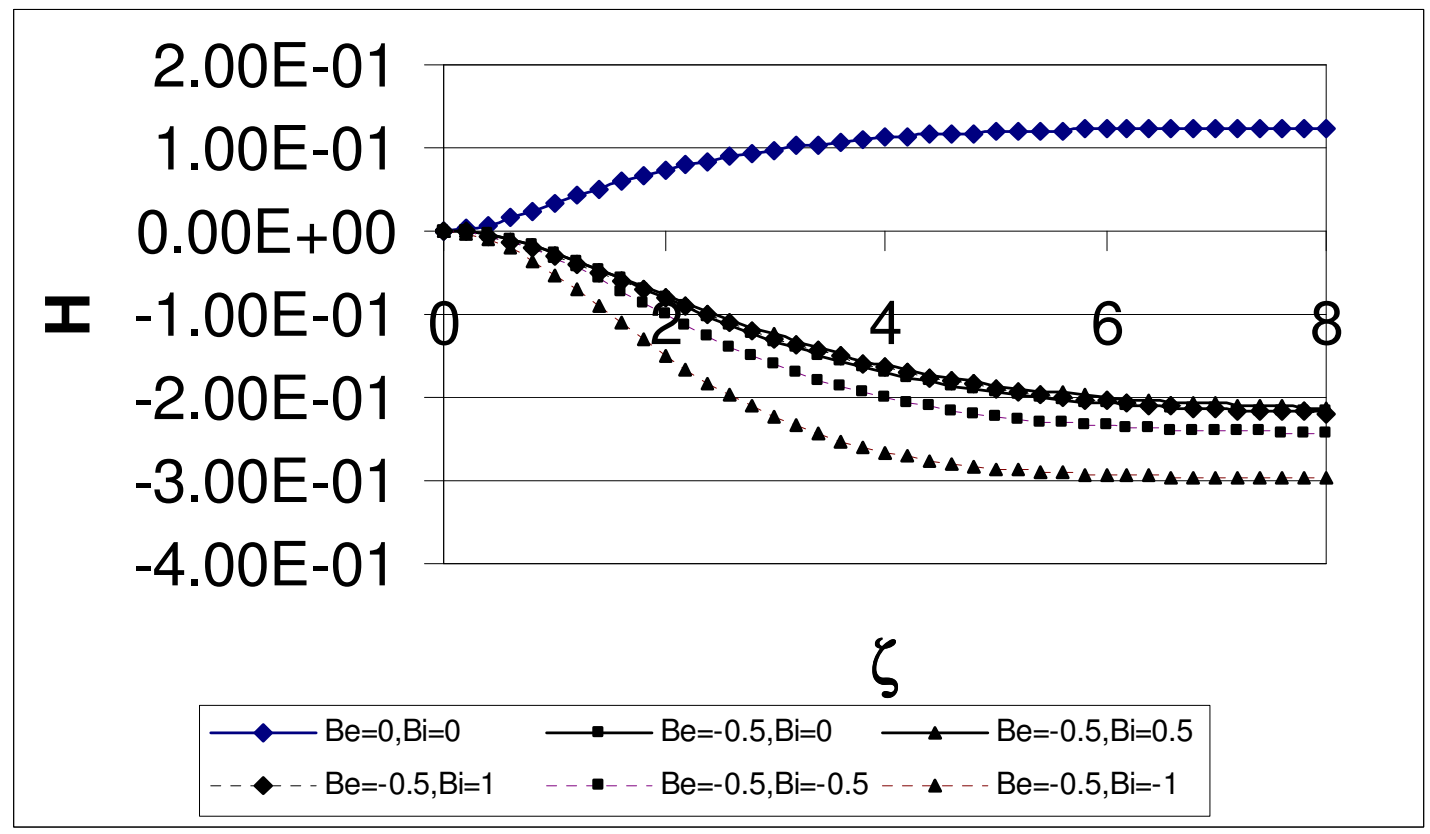

(d)

Fig. 3 Steady state profile of $H$ for various values of $B e, B i$ and $K$. (a) $K=0, B e \leq 0$; (b) $\mathrm{K}=0, \mathrm{Be} \geq 0$; (c) $\mathrm{K}=1, \mathrm{Be} \leq 0$; and (d) $\mathrm{K}=1, \mathrm{Be} \geq 0$. 


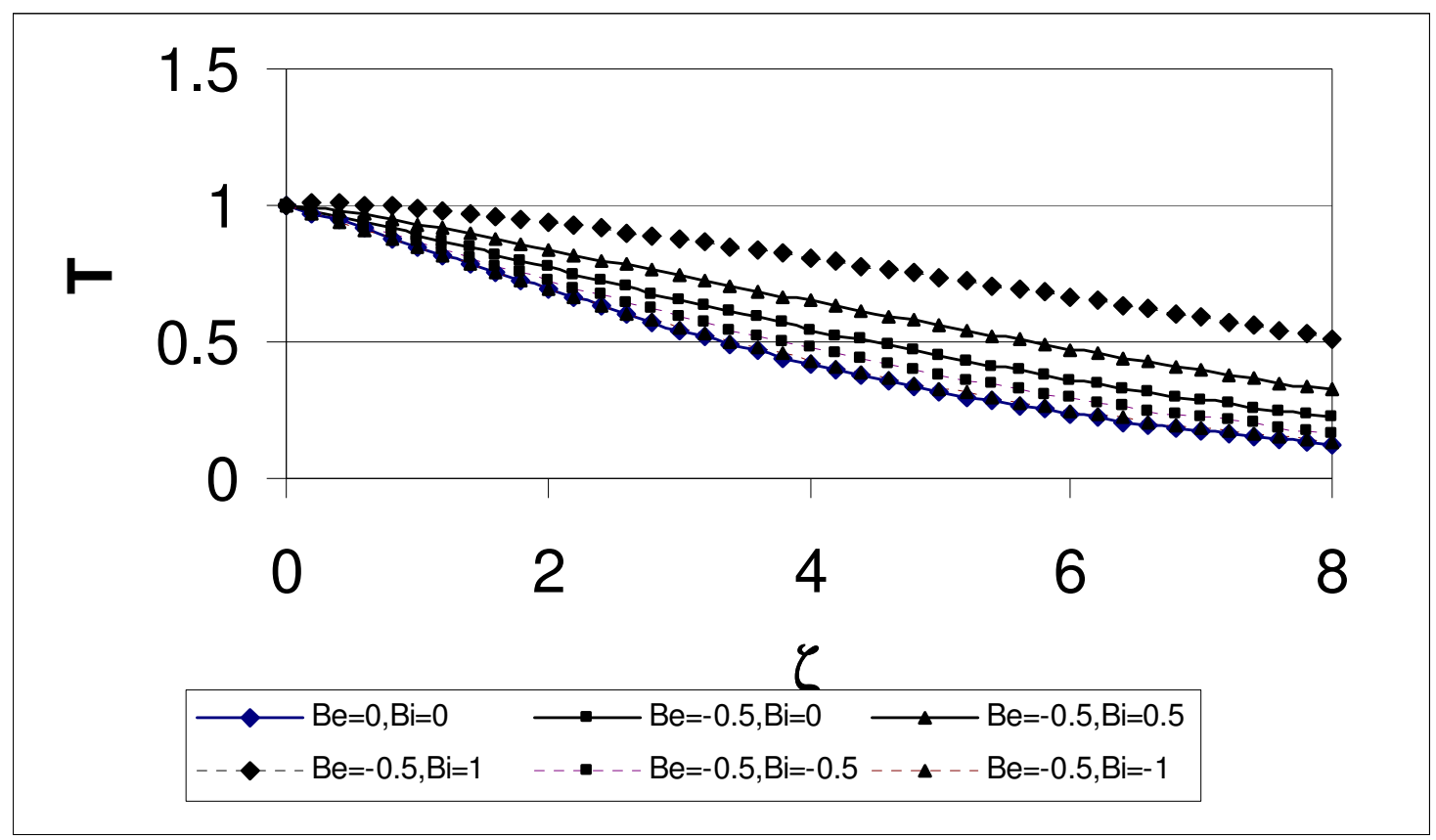

(a)

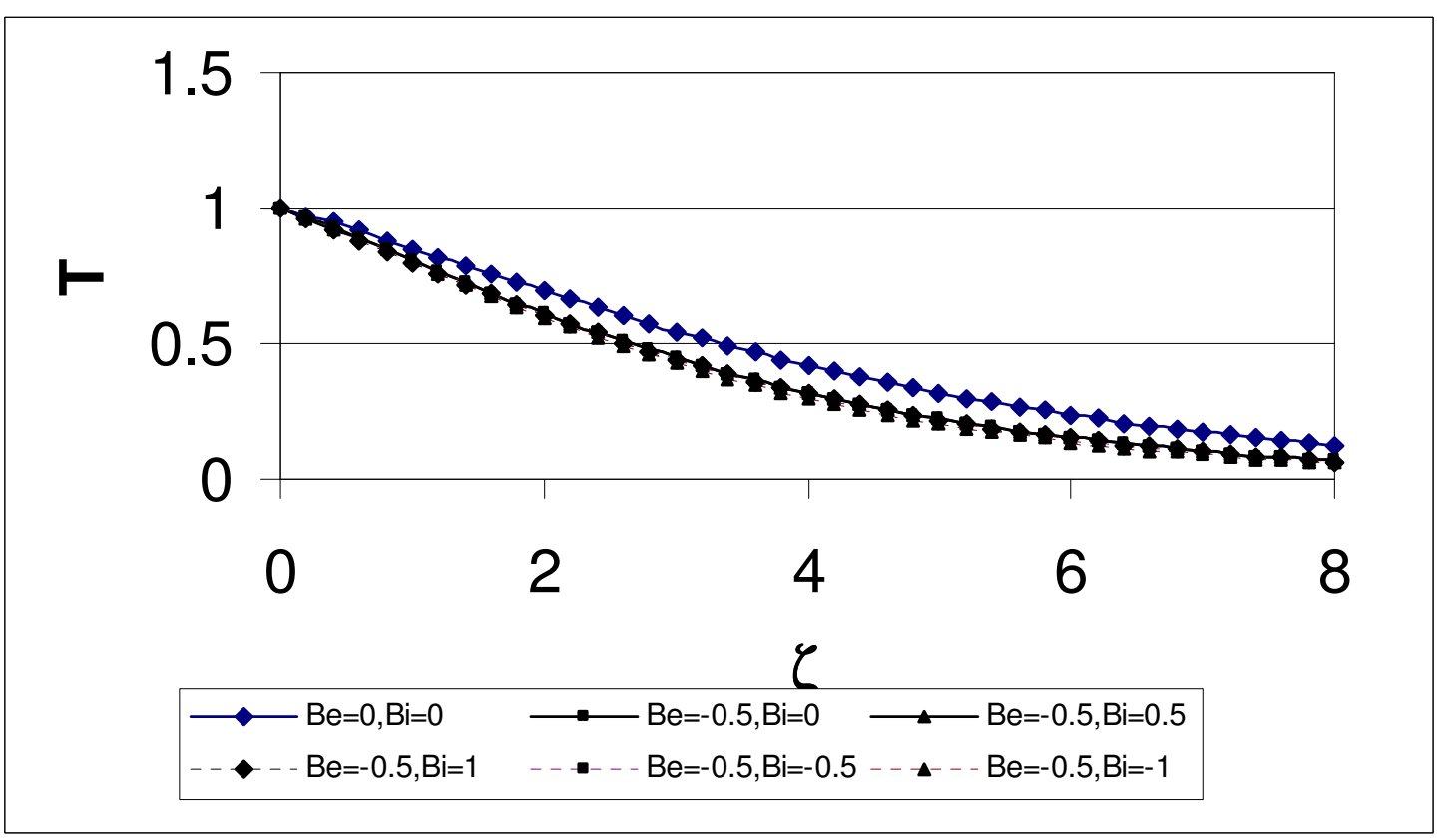

(b) 


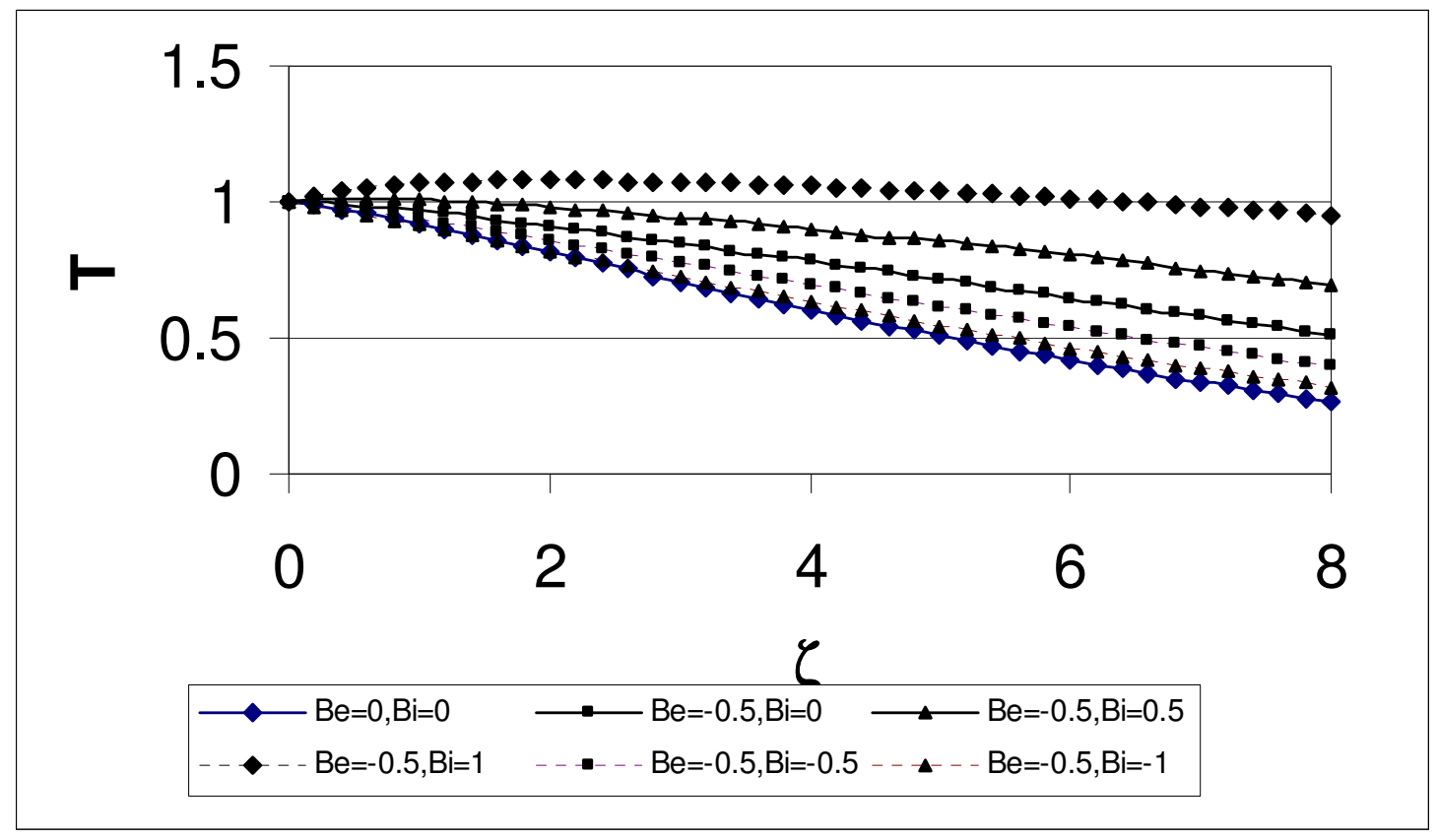

(c)

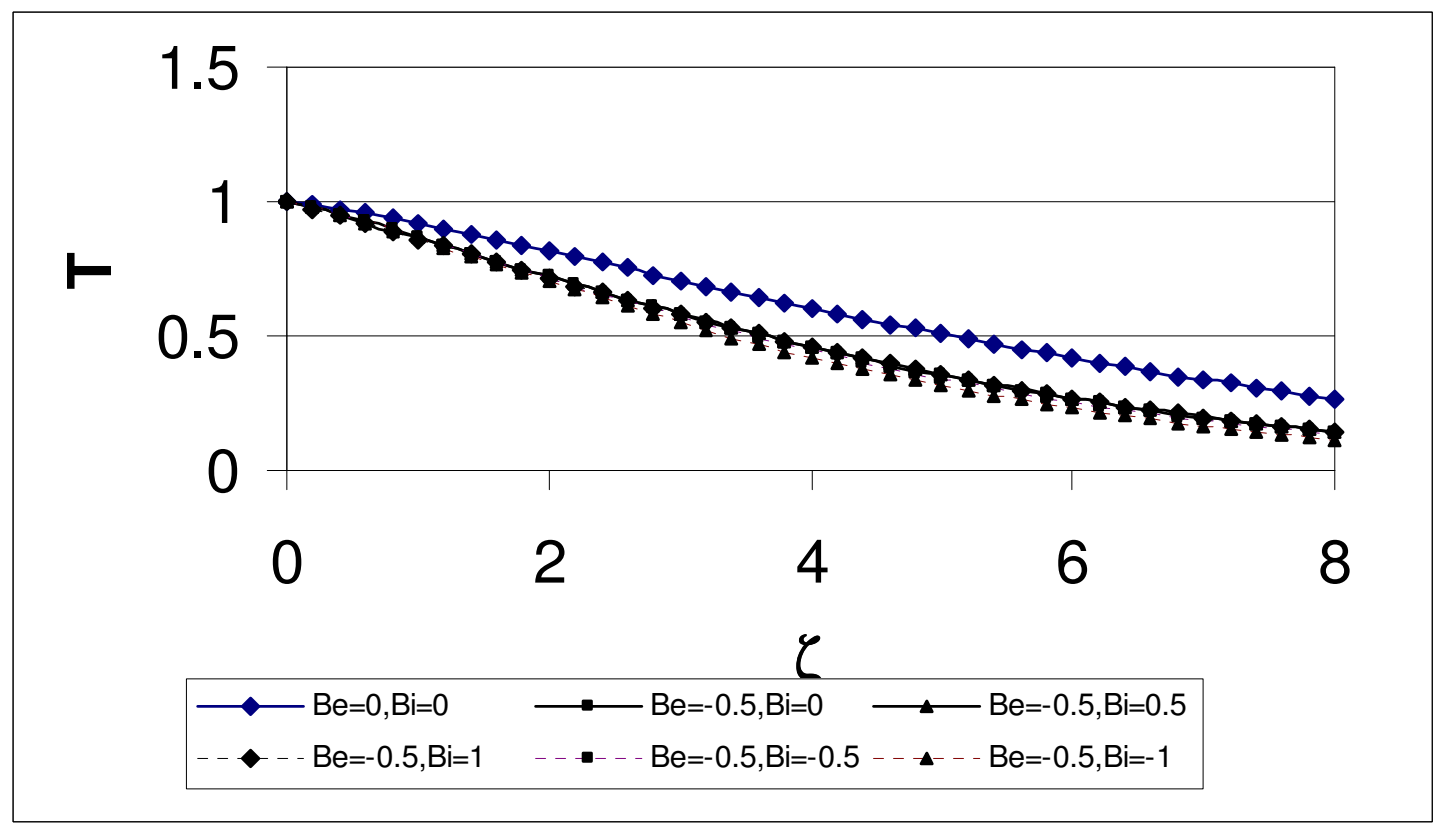

(d)

Fig. 4 Steady state profile of $T$ for various values of $B e, B i$ and $K$. (a) $K=0$, Bes $\leq 0$; (b) $\mathrm{K}=0, \mathrm{Be} \geq 0$; (c) $\mathrm{K}=1$, Be $\leq 0$; and (d) $\mathrm{K}=1, \mathrm{Be} \geq 0$. 
Table 1 Variation of the steady state value of $N_{u}$ with $B e, B i$ and $K$ for $P r=0.7$.

\begin{tabular}{|l|c|c|c|}
\hline$N_{u}$ & $K=0$ & $K=0.5$ & $K=1$ \\
\hline$B e=0, B i=0$ & 0.1646 & 0.1164 & 0.0729 \\
\hline$B e=-0.5, B i=0$ & 0.1136 & 0.0601 & 0.0158 \\
\hline$B e=-0.5, B i=0.5$ & 0.0474 & -0.0083 & -0.0543 \\
\hline$B e=-0.5, B i=1$ & -0.0556 & -0.1135 & -0.1636 \\
\hline$B e=-0.5, B i=-0.5$ & 0.1573 & 0.1061 & 0.0635 \\
\hline$B e=-0.5, B i=-1$ & 0.1875 & 0.1383 & 0.0973 \\
\hline$B e=0.5, B i=0$ & 0.2321 & 0.1877 & 0.1474 \\
\hline$B e=0.5, B i=0.5$ & 0.2425 & 0.1982 & 0.1587 \\
\hline$B e=0.5, B i=1$ & 0.2509 & 0.2069 & 0.1682 \\
\hline$B e=0.5, B i=-0.5$ & 0.2184 & 0.1743 & 0.1333 \\
\hline$B e=0.5, B i=-1$ & 0.1996 & 0.1564 & 0.1147 \\
\hline
\end{tabular}

Table 2 Variation of the steady state value of $N_{u}$ with $B e, B i$ and $K$ for $P r=10$.

\begin{tabular}{|l|c|c|c|}
\hline$N_{u}$ & $K=0$ & $K=0.5$ & $K=1$ \\
\hline$B e=0, B i=0$ & 0.2346 & -0.0119 & -0.2276 \\
\hline$B e=-0.5, B i=0$ & 0.1115 & -0.1204 & -0.2947 \\
\hline$B e=-0.5, B i=0.5$ & -0.3415 & -0.5891 & -0.7725 \\
\hline$B e=-0.5, B i=1$ & -1.1095 & -1.3574 & -1.5532 \\
\hline$B e=-0.5, B i=-0.5$ & 0.3841 & 0.1707 & 0.0066 \\
\hline$B e=-0.5, B i=-1$ & 0.5574 & 0.3601 & 0.2059 \\
\hline$B e=0.5, B i=0$ & 0.5659 & 0.3485 & 0.1410 \\
\hline$B e=0.5, B i=0.5$ & 0.6872 & 0.4839 & 0.2967 \\
\hline$B e=0.5, B i=1$ & 0.7702 & 0.5787 & 0.4069 \\
\hline$B e=0.5, B i=-0.5$ & 0.3791 & 0.1457 & 0.0888 \\
\hline$B e=0.5, B i=-1$ & 0.0827 & -0.1649 & -0.4338 \\
\hline
\end{tabular}

